school before completion in Bangladesh which negatively influences their mental well-being. The study adopted a quasi-experimental action evaluation strategy to investigate the role of an improved school landscape on children's subjective well-being. A Government primary school ground in Bangladesh was co-designed and developed which was used as a context and tool for formal teaching and informal play. The intervention included participatory design and development of the school ground, use of the school ground for learning of formal curriculum and also for informal play that took place between November 2014 and May 2015. Focus groups discussion were conducted with children $(n=29)$, teachers $(n=11)$ and parents $(n=6)$ both before and after the intervention in order to understand whether and how a designed environment can have an impact on children's teaching, learning and well-being. Semi-structured interviews were conducted with teachers $(n=2)$ who led children outdoors for teaching of science and mathematics. The findings suggest that the new schoolyard offered increased opportunities for exploration of the environment, physical activity and interaction with peers which positively influenced children's well-being.

\section{P19 THEMATIC ANALYSIS OF PLAYERS' REVIEWS OF VIRTUAL REALITY EXERGAMES}

${ }^{1}$ Nuša Farič, ${ }^{1}$ Henry Potts, ${ }^{2}$ Adrian Hon, ${ }^{3}$ Lee Smith, ${ }^{4}$ Katie Newby, ${ }^{1}$ Andrew Steptoe, ${ }^{1}$ Abi Fisher. ${ }^{1}$ University College London, London, UK; ${ }^{2}$ Six to Start, London, UK; ${ }^{3}$ Anglia Ruskin University, Cambridge, UK; ${ }^{4}$ Coventry University, Coventry, UK

\subsection{6/bmjopen-2019-QHRN.54}

Background Physical activity is associated with a variety of physical and psychosocial health benefits, but levels of moderate-to-vigorous intensity physical activity remain low worldwide. The application of virtual reality (VR) gaming systems involving movement (VR exergames) to promote physical activity is a promising avenue for engaging people in more physical activity. The aim of this study was to synthesise public reviews of popular VR exergames to identify common features that players like or disliked in order to inform future VR exergame design.

Methods We conducted a thematic analysis of 465 reviews of the 31 most popular exergames sold in the top three VR marketplaces, Steam, Viveport and Oculus. We identified the most common themes using thematic analysis.

Results The reviews were mixed, reporting a great variety of expectations, preferences and gaming experiences. Players preferred games that were highly realistic (e.g. closely simulate real-world sport), intuitive (in terms of the body movement and controls), and games that provided step-by-step increases in skill acquisition. Music was reported as greatly enhancing the experience. Favoured were social aspects with multiplayer options for company with friends or help from experienced players. Reviewers consistently reported that they felt VR exergaming was providing a high level of exertion equivalent to real world exercise, and the immersion/enjoyment was a welcome distraction from the exertion. There were three themes in negative reviews: the first was around bugs that rendered games frustrating. The quality of graphics had a particularly strong impact on perceived enjoyment. Reviewers disliked when games had overly complex controls and display functions that evoke motion sickness.
Conclusions VR exergames prove a stimulating way to engage in physical activity and a way to distract from negative perceptions of performing exercise. Future research calls for designing games that will stimulate the players in a realistic, intuitive, gradual step-wise way while meeting players' needs.

\section{P20 RATIONALITIES OF POSTPONING SCREENING: WOMEN'S INTERPRETATIONS OF CANCER RISKS AND PREVENTION}

${ }^{1}$ Gunvor Aasbø, ${ }^{2}$ Kari Nyheim Solbrække. ${ }^{1}$ Cancer registry of Norway, Oslo, Norge, Norway; ${ }^{2}$ Institute of Health and Society, University of Oslo, Oslo, Norway

\subsection{6/bmjopen-2019-QHRN.55}

Background In mainstream public health theory and praxis, citizens' ability to comply to healthy and preventative behaviour is often interpreted to be a question of knowledge. However, knowledge is complex, including a blend of scientific and medical knowledge, cultural attitudes, individual convictions and ignorance, in addition to personal uncertainties. Thus, in the context of cervical screening attendance, social constructions of knowledge play a pivotal role in the articulation of rationalities towards screening attendance.

Aim To generate a critical understanding of the social construction of knowledge about cervical cancer risks and prevention, in order to better understand why women in Norway may postpone cervical screening.

Methods We have completed 11 focus group interviews (FGI's) in Norway. In nine of the FGI, women who had postponed cervical screening participated. The FGI centred around six statements about cervical screening attendance.

Results Two important controversies were prominent in women's reflection about cervical cancer risk and prevention. First, cervical cancer was throughout the discussions understood as randomly acquired, but risk was modified by lifestyle, stress, genetics, and sexual risk behaviour. Secondly, women reflected conflicting views on the purpose of screening. Screening was mainly understood as diagnostic, and to less degree as prevention and for data-gathering purposes. In further analysis, we analyse how these different opinions are linked to attitudes of cancer fatalism and cancer fear, as well as cancer stigma and blame.

Conclusion Construction of knowledge of cancer risk and prevention has significance for understanding ambivalence regarding screening attendance in Norway.

\section{P21 'MY IDENTITY HAD JUST BEEN COMPLETELY DEVASTATED' - EXPERIENCES OF ADOLESCENT AND YOUNG ADULTS WITH}

${ }^{1}$ Ana Martins, ${ }^{2}$ Lindsey Bennister, ${ }^{1}$ Lorna Fern, ${ }^{3}$ Craig Gerrand, ${ }^{2}$ Maria Onasanya, ${ }^{4}$ Lesley Storey, ${ }^{5}$ Mary Wells, ${ }^{3}$ Julie Woodford, ${ }^{1}$ Rachael Windsor, ${ }^{1}$ Jeremy Whelan, ${ }^{1}$ Rachel Taylor. 'University College London Hospitals NHS Foundation Trust, London, UK; ${ }^{2}$ Patient Representative, UK; ${ }^{3}$ Royal National Orthopaedic Hospital, Stanmore, UK; ${ }^{4}$ Queens University Belfast, Belfast, UK; ${ }^{5}$ Imperial College Healthcare NHS Trust, London, UK

\subsection{6/bmjopen-2019-QHRN.56}

Background Treatment of sarcoma often involves long-term hospitalisation, extensive surgery, loss of mobility, complex rehabilitation programmes, and is in many cases accompanied by low expectations of survival. Subsequently, poorer patient- 
reported outcomes are recorded in comparison to patients with other cancer types. Studies examining psychosocial functioning of those affected by sarcoma are needed to improve wellbeing.

Aim This study aims to explore the experiences of adolescents and young adults (AYA) with sarcoma.

Methods A total of 35 AYA diagnosed with soft-tissue and bone sarcoma (19 female) aged 13-39 years old $(M=28.8$; $\mathrm{SD}=6.7)$ participated in semi-structured telephone/face-to-face interviews (25 diagnosed within the previous 4 years; 4 had an amputation). Interviews were recorded and transcribed verbatim and analysed using Framework analysis.

Results The quality of life domains of physical, emotional and social wellbeing were the overarching themes of analysis. In addition to descriptions of the impact of sarcoma on these domains, results showed three influencing factors of AYA experience: healthcare professionals' role; social support and coping strategies. AYA described experiences of being reassured and receiving information at the right level from professionals. Healthcare professionals' expertise and accessibility was valued by AYA. Family, friends and interactions with peers with cancer were core elements of support. Adaptation after diagnosis, treatment and/or surgery was influenced by the way AYA dealt with stress and adversity, with some struggling with finding their 'new normal'. Rehabilitation and therapy/counselling services had a considerable role in AYA's physical and emotional wellbeing; however access to these services was not equitable.

Conclusions A sarcoma diagnosis disrupts normality and initiates a process of re-examining expectations for self, daily life, and future hopes and plans. This study shows the impact of sarcoma on AYA's wellbeing and identity development; protective/risk factors identified could guide future support interventions for AYA with sarcoma.

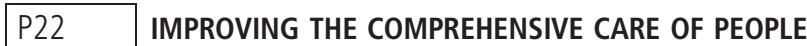 WITH ADVANCED CHRONIC DISEASES IN ACUTE HOSPITALS}

${ }^{1}$ Rosa Mertnoff, ${ }^{2}$ Cecilia Vindrola Padros, ${ }^{1}$ Eugenia Brague, ${ }^{3}$ Walter Cattaneo, ${ }^{1}$ Lucila Falcone, ${ }^{4}$ Maria Belen Ayala Torales, ${ }^{5}$ Mariangeles Pirchi. ${ }^{1}$ PALIATIVA NGO, Buenos Aires, Argentina; ${ }^{2}$ Department of Applied Health Research, UCL, London, UK; ${ }^{3}$ Hospital Central Dr. Ramón Madariaga, Posadas, Argentina; ${ }^{4}$ Hospital Dr. J.C Perrando, Resistencia Chaco, Argentina; ${ }^{5}$ Hospital José Ramón Vidal, Corrientes, Argentina

\subsection{6/bmjopen-2019-QHRN.57}

Background One of the most relevant challenges of healthcare systems is to provide high quality and cost-effective responses in progressive advanced chronic conditions with limited life prognosis and palliative care needs (PACC). These populations represent $75 \%$ of the mortality in middle-high income countries, a prevalence of $1.5 \%$ of general population, and $35 \%-45 \%$ of hospital admissions. Over the last 10 years, a new perspective of palliative care has emerged, identifying needs earlier and addressing them across all stages of patient's pathway. Our study is informed by this perspective and based on the implementation of a screening instrument identifying needs of PACC 48 hours after their hospital admission.

Objectives

1. Determine prevalence, clinical characteristics, and needs of PACC in acute care hospitals;
2. Identify barriers, difficulties, challenges and develop proposals to improve their attention;

3. Establish survival rates relating it to each illness trajectory (cancer, organ failure, frailty, and dementia).

Methods

Quantitative Phase:

Observational, multicenter, cross-sectional descriptive study to determine prevalence and clinical characteristics of PACC in 3 acute hospitals (AH) of the Northeast of Argentina (NEA) using NECPAL-CCOMS-ICO ${ }^{\circledR}$ instrument. It will involve a longitudinal analysis of a cohort of recruited patients for 1.2 years.

Qualitative Phase:

Each hospital will undergo a self-assessment survey exploring their current care delivery models and potential barriers they might face in delivering continuous care to PACC. Focus groups will be carried out with a sample of healthcare professionals exploring these barriers in greater detail.

Expected results

- Prevalence of PACC in AH

- Characteristics of PACC in AH of the NEA

- Survival of PACC identified at 1.2 years and predictive model

- Definition of improvement areas to elaborate an integrated assistance proposal, including training, quality standards and organizational changes adapting the use of hospital resources to implement a more efficient model of care delivery.

\section{P23 FRAGILE SUCCESS: FINDINGS FROM A REALIST PROCESS EVALUATION OF OPT-OUT BLOOD BORNE VIRUS TESTING}

${ }^{1}$ Seth Francis-Graham, ${ }^{1}$ Cecilia Vindrola, ${ }^{1}$ William Rosenberg, ${ }^{2}$ Tim Rhodes. ${ }^{1} U C L$, London, UK; ${ }^{2}$ London School of Hygiene and Tropical Medicine, London, UK

10.1136/bmjopen-2019-QHRN.58

The elimination of viral hepatitis C in England by 2025, is predicated on the diagnosis of blood-borne virus (BBV) infections in high prevalence settings, such as prison. In response to historic low testing rates, opt-out BBV testing has been implemented across the English estate. As part of a wider realist process evaluation, a high and low performing prison in London were selected for qualitative data generation, with the aim of unpicking conditions that facilitate or hinder programme performance.

Data were used to test context-mechanism-outcome theories. Four months were spent within each prison. Documentary analysis and unstructured observation captured aspects of the broader social and physical context. Semi-structured observation focused on the sequence of events that constitute opt-out testing, which was supplemented using realist interviews, conducted with prison officers, health workers, and patients.

Support from the Governor was crucial to ensure scarce resources were directed towards programme enablement. The testing was spearheaded by a single health worker and differences in personal motivation, experience, and confidence were salient. However, stress from pushing the programme, in an environment where healthcare was not prioritised, risked burnout. Within the poor performing 